\title{
Is cryptogenic stroke a tenable diagnosis? The Milpark Hospital patent foramen ovale closure experience
}

\section{Naomi Rapeport, Colin Schamroth and Nalin Patel}

Milpark Hospital, Johannesburg, South Africa

Address for correspondence:

Dr N. Rapeport

PO Box 84193

Greenside

2034

South Africa

Email:

nrapeport@global.co.za

\section{ABSTRACT Patent foramen ovale (PFO) is} increasingly being documented in patients presenting with "cryptogenic" neurological and peripheral ischemic events. Therapeutic options include the use of anti-platelet agents and in some cases non-surgical closure of the defect. In this article we present the Milpark Hospital experience of percutaneous closure of PFO to date. The importance of looking for this defect is highlighted, particularly in patients presenting with recurrent events despite antiplatelet therapy. Whilst routine closure of PFO is not advocated, it should be considered in certain select cases. SAHeart 2008; 5:96-100

Stroke is a syndrome encompassing a heterogeneous group of vascular diseases that lead to cerebral ischemia or hemorrhage. Conditions or factors that predispose to or increase the risk are diverse. Eighty-five percent of strokes are ischemic and most ischemic strokes occur in persons older than 65 years of age in association with the development of atherosclerosis. In contrast, younger patients with ischemic strokes often have few risk factors for atherosclerosis. (1) Approximately $40 \%$ of ischemic strokes have no clear etiology and are therefore termed cryptogenic. ${ }^{(2)}$ Transient ischemic attacks (TIAs) are a form of stroke, and should be regarded with the same degree of concern and importance as a stroke. TIAs are episodes of stroke symptoms that last only briefly, and the causes are similar to the causes of ischemic stroke. Infarcts of the brain occur in 15-50\% of TIAs even though neurological signs and symptoms are absent. ${ }^{(3)}$ The work-up of a patient presenting with a TIA or stroke should include routine laboratory tests (such as full blood count, urea and electrolytes, glucose, lipid profile, prothrombin index and a coagulation screen) as well as an ECG, chest X-ray, cerebral computed tomography or magnetic resonance imaging (MRI) of the brain, and ultrasound or magnetic resonance angiography of the carotid and vertebral arteries. If these studies do not identify the source of ischemia, then investigations directed specifically at detecting a cardiac cause are appropriate. Cardiac causes of stroke include atrial fibrillation, abnormalities of the left ventricle and left atrium, valvular lesions, and cardiac procedures. ${ }^{(4)}$ Detection of these latter problems involves the use of transthoracic or transoeophageal echocardiography (TEE), as well as ambulatory ECG monitoring for arrhythmias.

The role of patent foramen ovale (PFO) and atrial septal aneurysm (ASA) in the genesis of ischemic stroke in young adults has been investigated extensively over the past 20 years. ${ }^{(5,6,7)}$ Recently, the importance of PFO as an etiological factor for stroke or TIA in older patients has been highlighted. ${ }^{\left({ }^{(8)}\right.}$

Whilst patients with PFOs may present predominantly with neurological episodes, non-neurological events such as peripheral and pulmonary emboli may also occur.

The following report presents the Milpark Hospital experience with device closure of patent foramen ovale in 27 consecutive cases. The vascular event was considered the result of paradoxical embolism if the following conditions were met: presence of PFO with or without ASA with spontaneous or provocable (Valsalva maneuver) right-to-left shunting as assessed by TEE; clinically confirmed stroke/TIA or other vascular event; and exclusion of any other identifiable cause. 
CASES

Between November 2003 and March 2008, 27 patients underwent PFO closure at our centre. The demographic features of the patients are given in Table I. There were 12 males and 15 females with a mean age of 54.6 years. Eight patients had presented with a stroke and 12 patients with TIAs. TIA was defined as a focal neurological deficit resolving completely within 24 hours. Three patients presented with

TABLE I: Demographic features of patients

\begin{tabular}{|c|c|c|c|c|}
\hline Age & Gender & Presenting event & $\begin{array}{l}\text { TEE } \\
\text { findings }\end{array}$ & $\begin{array}{l}\text { Follow-up } \\
\text { (months) }\end{array}$ \\
\hline 38 & M & Stroke & PFO & 34 \\
\hline 50 & M & Multiple TIAs & PFO & 24 \\
\hline 45 & M & Multiple TIAs; initial TIA 4 years prior & PFO & 3 \\
\hline 50 & $\mathrm{~F}$ & Stroke & PFO & 8 \\
\hline 51 & $\mathrm{~F}$ & Stroke 10 years prior & PFO \& ASA & 14 \\
\hline 76 & $\mathrm{~F}$ & Embolus to hand & PFO & 6 \\
\hline 55 & $\mathrm{~F}$ & Atrial fibrillation & PFO & 24 \\
\hline 38 & M & $\begin{array}{l}\text { Coronary embolus: stroke } \\
14 \text { years prior }\end{array}$ & PFO & 13 \\
\hline 68 & M & $\begin{array}{l}\text { PE and history of multiple } \\
\text { cerebral emboli }\end{array}$ & PFO & 12 \\
\hline 31 & M & $\begin{array}{l}\text { TIA: coronary artery disease with } \\
\text { stent } 3 \text { years prior }\end{array}$ & PFO & 18 \\
\hline 58 & M & $\begin{array}{l}\text { Multiple TIAs: CT scan multiple } \\
\text { cerebral infarctions }\end{array}$ & PFO & 20 \\
\hline 39 & $\mathrm{~F}$ & $\begin{array}{l}\text { DVT, PE \& paradoxical embolism: } \\
\text { anti-phospholipid syndrome }\end{array}$ & PFO & 18 \\
\hline 51 & $\mathrm{~F}$ & TIA & PFO & 12 \\
\hline 49 & M & Stroke 10 years prior & PFO \& ASA & 15 \\
\hline 41 & $\mathrm{~F}$ & Stroke & PFO \& ASA & 10 \\
\hline 45 & $\mathrm{~F}$ & TIA & PFO & 7 \\
\hline 71 & $\mathrm{~F}$ & $\begin{array}{l}\text { TIA: stroke } 16 \text { years prior following } \\
\text { spinal surgery }\end{array}$ & PFO \& ASA & 11 \\
\hline 78 & $\mathrm{~F}$ & $\begin{array}{l}\text { Transient global amnesia: } \\
\text { TIA } 10 \text { years prior }\end{array}$ & PFO \& ASA & 10 \\
\hline 76 & $\mathrm{~F}$ & TIA & PFO \& ASA & 12 \\
\hline 46 & $\mathrm{~F}$ & Transient global amnesia & PFO & 2 \\
\hline 53 & M & Stroke & PFO & 2 \\
\hline 32 & $\mathrm{~F}$ & Stroke & PFO & 9 \\
\hline 67 & M & Retinal artery embolism & PFO & 5 \\
\hline 59 & M & Pulmonary embolism & PFO \& ASA & 3 \\
\hline 59 & M & TIA & PFO \& ASA & 2 \\
\hline 83 & $\mathrm{~F}$ & Transient global amnesia & PFO \& ASA & 1 \\
\hline 65 & $\mathrm{~F}$ & Multiple strokes & PFO \& ASA & 1 \\
\hline
\end{tabular}

deep vein thrombosis (DVT) and/or pulmonary embolism (PE) - one of whom had a history of multiple cerebral emboli that had not been investigated previously and another who had concomitant multiple strokes. Four patients presented with non-cerebral embolism. Of the 12 patients who presented with TIAs, 6 had a history of a previous neurological event (2 prior strokes and the others priorTIAs) that had occurred up to ten years prior to the presenting event. One patient, who presented with a coronary embolus, gave a history of a stroke 14 years earlier. Ten patients were on anti-platelet therapy prior to the presenting episode; 7 of them presented with TIA and 3 presented with non-cerebral embolism. It should be noted that the listed presenting event is not necessarily the indication for closure.

A coagulation screen was included in the work-up of these patients. Two were documented to have anti-phospholipid antibodies.

Seventeen patients had isolated PFOs and 10 patients had PFO with ASA. All device closures were with the Amplatzer PFO occluder (AGA Medical Corp, Plymouth, USA). The sizes used were $25 \mathrm{~mm}$ in 21 patients, $35 \mathrm{~mm}$ in 4 patients and $18 \mathrm{~mm}$ in 2 patients. All cases (with the exception of the first two) represent the experience of two operators (CS and NP). For patient comfort the procedures were performed under general anaesthesia, with $X$-ray screening and TEE guidance; with the exception of the last 2 cases, which were done under local anaesthesia utilising intracardiac ultrasound guidance. PFO closure was carried out through the femoral vein. A multipurpose catheter was passed across the PFO and sited in a pulmonary vein (preferably the left upper vein). A stiff guidewire was then inserted and the multipurpose catheter exchanged for a long sheath, the tip of which is placed in the left atrium just proximal to the point of entry into the pulmonary vein. The PFO occluder, attached to the delivery catheter, was then inserted and advanced to the tip of the catheter and the distal (left atrial) disc deployed immediately beyond the catheter tip. The device and sheath were then pulled back to allow the left atrial disc to align against the septum. The right atrial disc was then deployed. After checking for correct placement the device was released from the delivery catheter. Coronary angiography was frequently done at the same time, particularly in the older subjects to exclude concomitant coronary artery disease. Patients are given clopidogrel for one month and then continued with low dose aspirin. 
No patient has been lost to follow-up. Twenty-seven patients have been followed for a mean of I I months (range I-34 months). One patient died one year after device implantation. He had had multiple strokes and died from an intracerebral hemorrhage following a fall. No patient has reported a recurrent neurological or embolic event. Three patients reported palpitations after device closure (one case of atrial fibrillation, and two with symptomatic atrial extrasystoles). In all 3 cases the arrhythmias had resolved within 6 months.

\section{ILLUSTRATIVE EXAMPLES OF PATIENTS}

A 7I-year-old woman presented with recurrent TIAs. She had undergone spinal surgery sixteen years earlier.Two days postoperatively she suffered a stroke with infarction of the left parietal lobe confirmed on computed tomography. Cardiac assessment at that time, including a transthoracic echocardiogram, was normal. The event was diagnosed as a watershed stroke thought to be due to postoperative hypotension. Over time she developed a short ejection systolic murmur. Four years later she presented with her first TIA. Repeat transthoracic echocardiography documented mitral annular calcification and calcification of the aortic valve. She was treated with dipyridamole and aspirin. Thirteen years later she was reassessed by a neurologist following a TIA. She had a MRI scan of the brain which confirmed the previous left middle cerebral artery infarct. She was advised to have a further cardiac evaluation. She then underwent a TEE which demonstrated a PFO with an ASA, with spontaneous right-to-left shunting. The defect was closed and she has had no further TIAs. There have been several reports of paradoxical cerebral embolism occurring during spinal surgery in the presence of a PFO. ${ }^{9,10)} \mathrm{We}$ hypothesize that the original stroke was incorrectly ascribed to hypotension.

A 76-year-old woman had a history of hypertension. In 2001 she was diagnosed with borderline hypertension. Her workup included a transthoracic echocardiogram which was reported as normal. Five years later she was confirmed to have established hypertension and she was put onto anti-hypertensive therapy. A few months later she presented with an episode of confusion, subsequently diagnosed as an episode of transient global amnesia. She was investigated by a neurologist and an MRI scan performed and no specific abnormality was documented. She then underwent a TEE which demonstrated a PFO. The neurological episode was considered unrelated to the systemic hypertension, and more likely due to paradoxical embolism from the PFO.

An 83-year-old woman with established macular degeneration complained to her ophthalmologist of zigzag lines in front of her eyes. She had a history of longstanding obstructive pulmonary disease and systemic hypertension. The opthalmologist thought that the visual symptoms were not due to the macular degeneration and was concerned about the possibility of TIAs. She had carotid duplex studies which showed atherosclerotic disease but no significant ulceration or stenosis. She was placed on statins and aspirin and advised to come back for a TEE. She presented a few months later with an episode of transient global amnesia. On TEE a PFO was documented. This was closed and no further visual symptoms have occurred. Despite the presence of the "usual" risk factors for stroke/TIA and concomitant use of anti-platelet therapy, this patient had further episodes, prompting the search for another cause.

A 49-year-old man presented with a stroke in his early thirties and was diagnosed with neurological sarcoidosis. His carotid angiograms were normal but a transthoracic echo showed an ASA. He was anticoagulated and remained on warfarin therapy. He subsequently developed diabetes mellitus and both hypopituitarism and blindness as complications of the sarcoidosis. He also had a previous significant gastrointestinal bleed. He was deemed to be at high risk for continued warfarin therapy, prompting re-investigation of his ASA to establish whether a PFO was present. This in turn would allow for consideration of percutaneous closure, the discontinuation of anticoagulation and the use of low-dose aspirin as an alternative to warfarin. At TEE a PFO was documented in association with the ASA. The defect was subsequently closed percutaneously, and the warfarin therapy stopped.

One patient aged 46 years had been previously diagnosed with epilepsy and treated for this. She had had extensive neurological investigations, including brain scans and electroencephalograms, all of which were reported as normal. Despite anti-epileptic therapy she continued with neurological episodes. She had three episodes of transient global amnesia, which warranted a referral for a disability medical evaluation. Assessment with TEE showed a PFO. At the time of closure, coronary angiography revealed a sub-total occlusion of the right coronary artery. This was not an anticipated finding and the patient had not undergone formal assessment for ischemia prior to the PFO closure procedure. It 
was decided to intervene on the stenosis empirically, and a coronary stent was inserted. Post procedure the patient confirmed that she had been experiencing episodes of angina-like chest pain.

A 31 -year-old male had presented with a myocardial infarction at the age of 26 years that was thought to relate to the use of anabolic steroids. At that time, a right coronary artery stenosis was treated with stenting. He had been compliant with therapy including aspirin and statins. He subsequently presented to a neurologist complaining of headaches. A diagnosis of migraine was made, based upon his description of altered vision, which was thought to be a migraine aura. On closer questioning it was evident that these were episodes of amaurosis fugax. ATEE showed a PFO which was closed and no further TIAs (or migraines) have occurred.

\section{DISCUSSION}

The presence of a patent foramen ovale (PFO) has been shown to be associated with an increased risk of ischemic stroke. ${ }^{(5,6,7)}$ While this association has been predominantly shown in younger patients with unexplained (cryptogenic) stroke, it has also been suggested to be a factor in older patients. $(5,8,11,12)$

Patent foramen ovale is associated with right to left shunting. The foramen ovale remains open in about one-fourth of the general population. The prevalence decreases with increasing age, from 34\% during the first three decades to $20 \%$ during the ninth decade. ${ }^{(13)}$ The risk of paradoxical embolism increases with increasing size of PFO. With each decade of life the size of the PFO increases, so that the mean diameter in the first decade is $3.4 \mathrm{~mm}$, whilst in the tenth decade the mean size is $5.8 \mathrm{~mm} .^{(12,13)}$

The role of PFO in the pathogenesis of ischemic stroke was suggested by a case controlled study in patients under 55 years of age with ischemic stroke detected by contrast echocardiographic examinations. ${ }^{(5)}$ The prevalence of PFO was significantly higher in the stroke group than in controls (40\% vs. 10\%; $p<0.001$ ). Patent foramen ovale was found in $54 \%$ of stroke patients with no other identifiable cause. This study concluded that PFO associated paradoxical embolism was the cause of stroke. The subsequent PFO-ASA Study supported these conclusions, finding that $46 \%$ of young cryptogenic stroke patients had PFO. ${ }^{(14)}$ It appears that patients with PFO have an increased prevalence of pelvic vein thrombosis. ${ }^{(15)}$ In this study of young patients with cryptogenic stroke, pelvic vein thrombosis was more prevalent than in controls (20\% vs. 4\%). This may be the true source of the embolus with the PFO being the conduit that allows the embolus to cross to the systemic circulation.

A prospective study of 598 patients (ages 18 to 55 years) presenting with cryptogenic stroke showed that $36 \%$ had a PFO, 1.7\% had an ASA, and $8.5 \%$ had both abnormalities. Patients with both PFO and ASA who have had a stroke are at higher risk for recurrent stroke despite continued therapy with aspirin. ${ }^{(6,16)}$ These studies excluded patients over the age of 55 years due to the higher prevalence of large vessel atherosclerosis in this age group. The association of PFO with cryptogenic stroke in older patients (those above 55 years) has only recently been reported. ${ }^{(8)}$ Handke et al. have reported on the importance of PFO as an etiological factor in cryptogenic stroke in older patients. Their recent prospective study of patients with cryptogenic stoke compared with patients with stroke of known cause, looked at the prevalence of PFO with or without an associated ASA using transoesophageal echocardiography. They found that the prevalence of PFO was significantly greater among patients with cryptogenic stroke than among those with stroke of known cause for both younger (43.9\% vs. 14.3\%) and older patients (28.3\% vs. II.9\%) respectively. The association was more pronounced among patients with both PFO and ASA in both age groups (I 3.4\% vs. $2.0 \%$ in younger subjects and $15.2 \%$ vs. $4.4 \%$ in older subjects). Multivariate analysis showed that the presence of PFO was independently associated with cryptogenic stroke in both age groups with odds ratio of 3.70 in the younger group and odds ratio of 3.00 in the older group.

Therapeutic options for patients with PFO with or without ASA, who present with a stroke or TIA include antiplatelet therapy with aspirin, dipyridimole or clopidogrel, oral anti-coagulation with warfarin, or closure of the defect surgically or percutaneously. Percutaneous closure of the defect is a well established procedure with a low complication rate in skilled hands. It is effective and associated with a lower rate of recurrent neurological events than medical therapy alone. This has been confirmed in several observational studies and meta-analyses. ${ }^{(17,18,19,20,21)}$

Our reported series demonstrates the importance of taking an adequate history, and not always accepting an "obvious" pathology as the cause of the neurological deficit or vascular event. These cases emphasise the need to reassess patients, particularly when symptoms 
occur despite apparent appropriate therapy. None of the cases had a PFO closed merely because of its presence. All patients had neurological symptoms or other embolic complications, many despite being on antiplatelet therapy or oral anticoagulants. Most of the patients had recurrent events, often many years after the initial one. The term cryptogenic implies "hidden source", a cause is not found. Although the link between TIA/Stroke and PFO/ASA is highly suggestive but still largely unproven, we suggest that the term "cryptogenic" only be considered after a TEE has been performed and a PFO, with or without an ASA, is ruled out. Long-term follow-up of a patient cohort like the one presented could give added support to such linkage. The work-up of a patient with TIA or stroke cannot be said to be complete without the performance of a TEE. Whilst this may be more imperative in the younger patient, older subjects should not be excluded.

\section{ACKNOWLEDGEMENTS}

The authors would like to acknowledge the assistance of Dr J. Harrisberg with the first two cases.

\section{REFERENCES:}

I. Kristensen B, Malm J, Carlberg B, et al. Epidemiology and etiology of ischemic stroke in young adults aged 18 to 44 years in northern Sweden. Stroke 1997;28:1702-1709.

2. Leys D, Bandu L, Henon H, et al. Clinical outcome in 287 consecutive young adults ( 15 to 45 years) with ischemic stroke. Neurology 2002;59:26-33.

3. Smith WS, English JD, Johnston SC. Cerebrovascular diseases. Harrison's principles of internal medicine. McGraw Hill Medical, 17th Ed. 2008;2521.

4. Adams HP, del Zoppo G], von Kummer R. Management of Stroke: a practical guide for the prevention evaluation and treatment of acute stroke. 2000. Professional Communications, Inc. USA.

5. Lechat P, Mas JL, Lascault G, et al. Prevalence of patent foramen ovale in patients with stroke. N Engl | Med 1988;3 | 8:1 | 488-1 | 52.

6. Overell JR, Bone I, Lees KR. Interatrial septal abnormalities and stroke: a meta-analysis of case-control studies. Neurology 2000;55: | 172-1 179.

7. Webster MW, Chancellor AM, Smith HJ, et al. Patent foramen ovale in young stroke patients. Lancet 1988;2:1 I-12.

8. Handke M, Harloff A, Olschewski M, et al. Patent foramen ovale and cryptogenic stroke in older patients. N Engl J Med 2007;357:2262-2268.

9. Horlocker TT, Wedel DJ, Cucchiara RF. Venous air embolism during spinal instrumentation and fusion in the prone position. Anesth Analg 1992;75: 152.

10. Rodriguez RA, Sinclair B, Weatherdon D, Letts M. Patent foramen ovale and brain microembolization during scoliosis surgery in adolescents. Spine 200 I;26:1719-1721.

1 I. Di Tullio M, Sacco RL, Gopal A, et al. Patent foramen ovale as a risk factor for cryptogenic stroke. Ann Intern Med 1992; | 17:461-465.

12. Hausmann D, Mügge A, Daniel WG. Identification of patent foramen ovale permitting paradoxic embolism. J Am Coll Cardiol 1995;26: 1030-1038.

13. Hagen PT, Scholz DG, Edwards WD. Incidence and size of patent foramen ovale during the first 10 decades of life: an autopsy study of 965 normal hearts. Mayo Clin Proc 1984;59:17-20.

14. Lamy C, Giannesini C, Zuber M, et al. Clinical and imaging findings in cryptogenic stroke patients with and without patent foramen ovale: the PFO-ASA Study. Atrial Septal Aneurysm. Stroke 2002;33:706-7।I.

15. Cramer SC, Rordorf G, Maki JH, et al. Increased pelvic vein thrombi in cryptogenic stroke: results of the Paradoxical Emboli from Large Veins in Ischemic Stroke (PELVIS) study. Stroke 2004;35:46-50.

16. Mas JL, Arquizan C, Lamy C, et al. Recurrent cerebrovascular events associated with patent foramen ovale, atrial septal aneurysm, or both. N Engl J Med 200I; 345: 1740- 1746.

17. Windecker S, Wahl A, Nedeltchev K, et al. Comparison of medical treatment with percutaneous closure of patent foramen ovale in patients with cryptogenic stroke. J Am Coll Cardiol 2004;44:750-758.

18. Wu LA, Malouf JF, Dearani JA, et al. Patent foramen ovale in cryptogenic stroke: Current understanding and management options. Arch Intern Med 2004; | 64:950-956.

19. Slavin L, Tobis JM, Rangarajan K, et al. Five-year experience with percutaneous closure of patent foramen ovale. Am J Cardiol 2007;99:1316-1320.

20. Thanopoulos BV, Dardas PD, Karanasios E and Mezilis N. Transcatheter closure versus medical therapy of patent foramen ovale and cryptogenic stroke. Catheter Cardiovasc Interv 2006;68:741-746

21. Onorato E, Melzi G, Casilli F, et al. Patent foramen ovale with paradoxical embolism: Mid-term results of transcatheter closure in 256 patients. J Interv Cardiol 2003: 16:43-50. 